\title{
Erratum
}

Vet. Res. 32 (2001) 275-284

\section{Quinolone resistance in Escherichia coli}

\author{
Mark WebBer, Laura J.V. PIDDOCK* \\ Antimicrobial Agents Research Group, Division of Immunity and Infection, \\ The Medical School, University of Birmingham, Birmingham, B15 2TT, United Kingdom
}

Dr L. Piddock wishes to modify the text of her article, page 282, third paragraph, lines 5-7 of the second column:

"most of this decrease is due to the withdrawal of enrofloxacin from use in feed and water" should be

"most of this decrease is due to the withdrawal of enrofloxacin from use in feed for medication of pigs." 